\title{
On Prime Labeling of some Classes of Graphs
}

\author{
N. Ramya \\ Dept.of. Maths, \\ Bharath University, Selaiyur, \\ Chennai-73.
}

\author{
K. Rangarajan \\ Dept.of. Maths, \\ Bharath University, Selaiyur, \\ Chennai-73.
}

\author{
R. Sattanathan \\ Dept.of.Maths, \\ D.G.Vaishnav College, \\ Chennai-106.
}

\begin{abstract}
Graph labeling is an important area of research in Graph theory. There are many kinds of graph labeling such as Graceful labeling, Magic labeling, Prime labeling, and other different labeling techniques.In this paper the Prime labeling of certain classes of graphs are discussed.It is of interest to note that $\mathrm{H}$-graph which is a 3 -regular graph satisfy Prime labeling. A Gear graph is a graph obtained from Wheel graph, with a vertex added between each pair of adjacent vertices of an outer cycle. It is proved in general this graph is Prime. Yet another class of graphs is the Sun flower graph and corona of Cycle graph $C_{n}$ and $K_{1,3}$.A stepwise algorithm is given to prove that both these classes of graphs satisfy Prime labeling.
\end{abstract}

\section{Keywords}

Prime Labeling, H-Graph, Gear Graph, Sunflower Graph, Corona of $\mathrm{C}_{\mathrm{n}}$ and $\mathrm{K}_{1,3}$

\section{INTRODUCTION}

Labeling of a graph $\mathrm{G}$ is an assignment of integers either to the vertices or edges or both subject to certain conditions $[2,3]$. A dynamic survey on graph labeling is regularly updated by Gallian [1] and it is published in electronic journal of combinatorics. The different kinds of the graphs are studied by us are found in $[5,7,8]$. A Graph $G=G(V, E)$ with $V$ vertices is said to admit prime labeling if its vertices can be labeled with distinct positive integers not to exceeding $\mathrm{V}$ such that the labels of each pair of adjacent vertices are relatively prime. A graph $\mathrm{G}$ which admits prime labeling is called a prime graph [6].

\section{PRELIMINARIES AND NOTATIONS}

In this section, we give the basic definitions relevant to this paper. We always denote Let $\mathrm{G}=\mathrm{G}(\mathrm{V}, \mathrm{E})$ to be a finite, simple and undirected graph with ' $\mathrm{V}$ ' vertices and " $\mathrm{E}$ “edges.

\section{Definition 1:}

Let $\mathrm{G}=\mathrm{G}(\mathrm{V}, \mathrm{E})$ be a graph. A bijection $\mathrm{f}: \mathrm{V} \rightarrow\{1,2, \ldots \ldots . . \mathrm{v} \mid\}$ is called prime labeling if for each $e=\{u, v\}$ belongs to $E$, we have GCD $(\mathrm{f}(\mathrm{u}), \mathrm{f}(\mathrm{v}))=1$. A graph that admits a prime labeling is called prime graph.

\section{Definition 2:}

An $\mathrm{H}$ graph $\mathrm{H}(\mathrm{r})$ [4],[10] is a 3-regular graph with vertex set $\{(\mathrm{i}, \mathrm{j}): 1 \leq \mathrm{i} \leq 3 ; 1 \leq \mathrm{j} \leq 2 \mathrm{r}\}$ and edge set $\{(\mathrm{i}, \mathrm{j}),(\mathrm{i}, \mathrm{j}+1) \mathrm{i}=1,3$, $1 \leq \mathrm{j} \leq 2 \mathrm{r}-1\}$ u $\{((2, \mathrm{j}),(2, \mathrm{j}+1)), \mathrm{j}$ odd, $1 \leq \mathrm{j} \leq 2 \mathrm{r}-1\}$ $\mathrm{u}\{((1,1),(1, \mathrm{n}),(3,1),(3, \mathrm{n}))\} \mathrm{u}\{(\mathrm{i}, \mathrm{j}),(\mathrm{i}+1, \mathrm{j}+1) \mathrm{i}, \mathrm{I}=1,2,1 \leq \mathrm{j} \leq 2 \mathrm{r}$.

An $\mathrm{H}$ graph $\mathrm{H}(\mathrm{r})$ has 6r vertices and 9r edges .

\section{Definition 3:}

Gear graph $\mathrm{G}_{\mathrm{r}},[4]$ also known as a bipartite wheel graph is a wheel graph with a vertex added between each pair of adjacent vertices of the outer cycle. Gear graph $G_{r}$ has $2 r+1$ vertices and $3 r$ edges.

\section{Definition 4:}

The Helm $\mathrm{H}_{n}[9]$, is the graph obtained from a wheel by attaching a pendant edge at each vertex of the $n$-cycle.

\section{Definition 5:}

A Flower is the graph obtained from a Helmgraph by joining each pendant vertex to the central vertex of the helm graph.

\section{Definition 6:}

The Sun flower graph $\mathrm{V}[\mathrm{n}, \mathrm{s}, \mathrm{t}]$ is the resultant graph obtained from the flower graph of wheels $\mathrm{W}_{\mathrm{n}}$ by adding $\mathrm{n}-1$ pendant edges to the central vertex.

We construct $V[n, s, t]$ as follows,

Consider the wheel graph $W_{n}$ with ' $n$ ' vertices and 2(n-1) edges. It is the graph on ' $\mathrm{n}$ ' vertices constructed by connecting a single vertex to every vertex in an (n-1) cycle. Then by attaching a pendant edge at each vertex of the n-cycle we get an helm graph with $2 n-1$ vertices and $3(n-1)$ edges. Now by joining each pendant vertex to the central vertex of the helm we get a flower graph with the same $2 n-1$ vertices and $4(n-1)$ edges. Finally, by adding n-1 pendant edges to the central vertex of the wheel we obtain the sunflower graph $\mathrm{V}[\mathrm{n}, \mathrm{s}, \mathrm{t}]$ with $\mathrm{s}=(3 \mathrm{n}-2)$ vertices and $\mathrm{t}=5(\mathrm{n}-1)$ edges.

\section{Definition 7:}

The graph corona of $\mathrm{C}_{\mathrm{n}}$ and $\mathrm{k}_{1,3}$ is obtained from a cycle $\mathrm{C}_{\mathrm{n}}$ by introducing ' 3 ' new pendant edges at each vertex of cycle.

\section{MAIN RESULTS}

\section{Theorem 1:}

An $\mathrm{H}$-graph $\mathrm{H}(\mathrm{r})$ is a 3-regular graph has 6r vertices and $9 \mathrm{r}$ edges. We prove $\mathrm{H}(\mathrm{r})$ is prime. We give below an algorithm of how to label H(r).So that it become prime.

Proof:

Step 1:

The prime labeling of $\mathrm{H}(1)$ is as follows;

The right arm of the $\mathrm{H}(1)$ can be labeled as in clockwise direction 1,2,3and left arm of the $\mathrm{H}(1)$ can be labeled as 4,5,6

\section{Step 2:}

We labeled $\mathrm{H}(1)$ as given in step 1 and $\mathrm{H}(2)$ is labeled as follows. The right arm of the $\mathrm{H}(2)$ can be labeled as in clockwise direction 7,8,9 and left arm of $\mathrm{H}(2)$ is $10,11,12$ as shown in the following figure : 


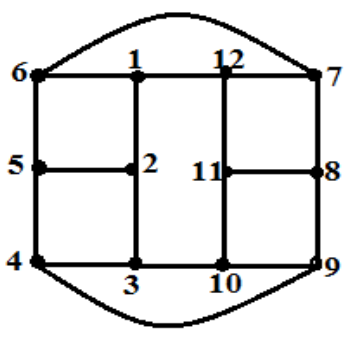

Step 3:

W have to be labeled $\mathrm{H}(1), \mathrm{H}(2), \ldots \ldots$ as given in step 1 and step 2 and finally $\mathrm{H}(\mathrm{r})$ is labeled as in clockwise direction 6r$5,6 r-2,6 r-1,6 r$ as shown in the following figure.

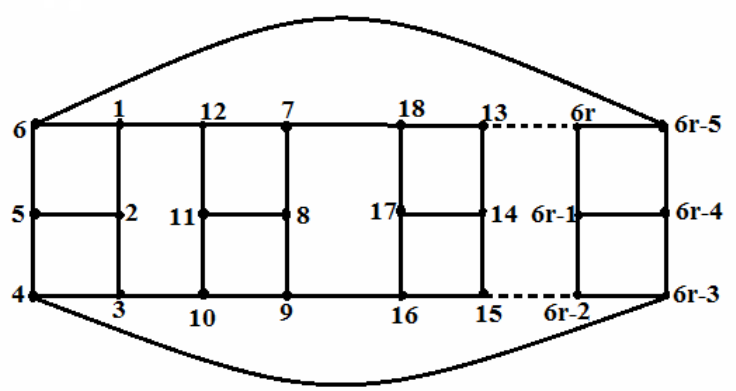

As seen by the algorithm the following pairs(6r,6r-5), (6r-1,6r-4), (6r-2,6r-3), (6r,6r-1), (6r-1,6r-2), (6r-5,6r-4), $(6 r-4,6 r-3),(6 r-5,6),(6 r-3,4)$ are always prime, when $1 \leq r \leq 9$.

\section{Theorem 2:}

A Gear graph $G_{r}, r \geq 3$, there exists a $(2 r+1)$ vertices and $3 r$ edges we prove $\mathrm{G}_{\mathrm{r}}$ is prime.

\section{Proof:}

Step 1: The central vertex has to be labeled as 1 .

Step 2: Rest of the vertices in the outer cycle has to be labeled as $2,3, \ldots . . n-1$ where ' $n$ ' be the number of vertices

\section{Example Gear graphG 6}

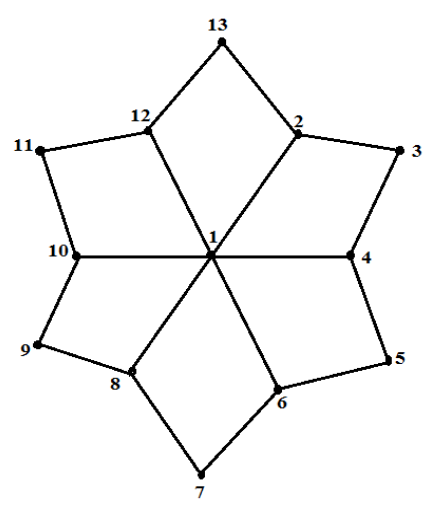

\section{Theorem 3:}

If (n-1) pendant vertices are attached at the central vertex of a flower graph of wheel $\mathrm{W}_{\mathrm{n}}$ for $\mathrm{n}=5,7,9 \ldots$ then we get sunflower graph having $\mathrm{s}$ vertices and $\mathrm{t}$ edges then the resulting graph $\mathrm{V}[\mathrm{n}, \mathrm{s}, \mathrm{t}$,$] is prime.$

\section{Proof:}

Step 1: The central vertex has to be labeled as 1 .
Step2: Number of vertices on the wheel is equal to the number of pendant vertices (n-1) pendant vertices are attached with the central vertex. It has to be labeled as $2,3, \ldots . n$.

Step3: The prime labeling of $\mathrm{W}_{\mathrm{n}}$ is given by $\mathrm{n}+2, \mathrm{n}+4, \ldots \ldots \mathrm{n}+\mathrm{x}$.

When $\mathrm{n}=5,7,9 \ldots . \mathrm{x}$ will be $8,12,16 \ldots$ respectively

Step4: The rest of the vertices of the flower graph will be labeled as $\mathrm{n}+1, \mathrm{n}+3, \mathrm{n}+5 \ldots \mathrm{n}+\mathrm{y}$ when $\mathrm{n}=5,7,9 \ldots \mathrm{y}$ will be $7,11,15$ respectively.

Example: Sunflowergraph V[8,s,t]

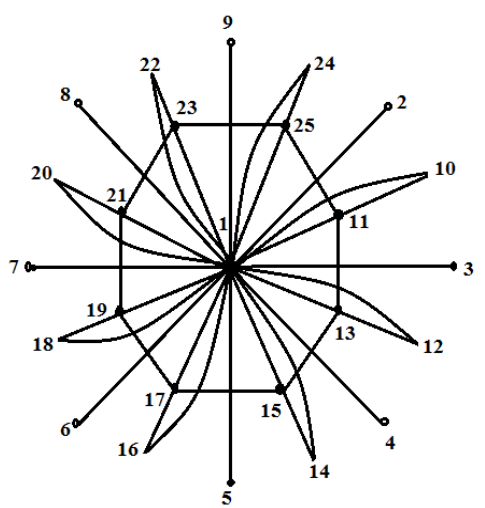

Theorem 4:

We prove corona of $C_{n}(n \geq 3)$ and $k_{, 1,3}$ is prime.

Proof:

Prime labeling of $\mathrm{C}_{\mathrm{n}}$, will be classified in to following sub cases:

Case i)

When $n=3$, and $n=4$ these cases are discussed separately.

In $\mathrm{C}_{\mathrm{n}}$ When $\mathrm{n}=3$, has to be labeled as $1,2,3$. In $\mathrm{k}_{1,3}$ the labeling of pendant vertices joined with even number ' 2 ' will be labeled as odd numbers 5,7,9 satisfying the condition of prime labeling. Another labeling of pendant vertices joined with odd number 3 will be labeled as even numbers $4,8,10$. Rest of the numbers $6,11,12$ has to be labeled in the pendant vertices that should be joined with 1 .

In $\mathrm{C}_{\mathrm{n}}$ when $\mathrm{n}=4$, has to be labeled as $1,2,3,4$.In $\mathrm{k}_{1,3}$ the labeling of pendant vertices joined with even number 2 will be labeled as odd number 5,7,9 satisfying the prime labeling condition. Another labeling of pendant vertices joined with odd number 3 will be labeled as 8,10,14. The labeling of pendant vertices joined with even number 4 will be labeled as odd number $11,13,15$. Rest of the numbers $6,12,16$ has to be labeled in the pendant vertices that should be joined with 1 .

Case ii)

(i) In $\mathrm{C}_{\mathrm{n}}$ when $\mathrm{n}=5$, has to be labeled as $1,2,3,4,5$.

(ii) In $C_{n}$ (when $n=6,7,8,9,10,11$ ) has to be labeled as $1,2 \ldots . . n$ excluding 6 .

(iii) In $\mathrm{C}_{\mathrm{n}}$ [when $\left.\mathrm{n}=12,13,14,15,16,17\right]$ has to be labeled from $1,2 \ldots n$ excluding 6,12 . 
(iv) Generally in $C_{n}(n \geq 5)$ the labeling of vertices with multiples of 6 are prohibited other integers are occur in order

Case iii)

In $\mathrm{k}_{1,3}$ the labeling of pendant vertices joined with even number will be labeled as odd number satisfying the condition of prime labeling.

Case iv)

The labeling of pendant vertices joined with odd number will be labeled as even number satisfying the prime labeling conditions.

\section{Case v)}

The $\mathrm{k}_{1,3}$ branch vertices which lies on the cycle $\mathrm{C}_{\mathrm{n}}$ prime labeled vertex 5 , has to be labeled as $6,12,18$.

\section{Case vi)}

Similarly the $\mathrm{k}_{1,3}$ branch vertices which lies on the cycle $\mathrm{C}_{\mathrm{n}}$ with prime labeled vertex 7 has to be labeled as 24,30,36 provided the total number of vertices exceed 36 , otherwise we follow case iv) similarly we continue this process for the vertices $11,13,17, \ldots$.

Case vii)

Rest of the numbers has to be labeled in the pendant vertices that should be joined with 1 .

Example: Corona of $\mathrm{C}_{6}$ and $\mathrm{k}_{1,3}$

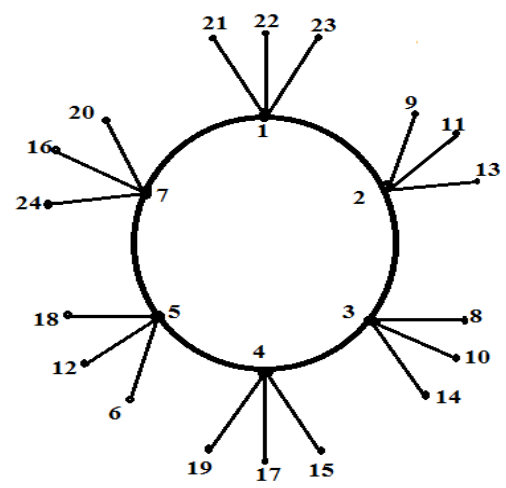

\section{CONCLUSION}

We have presented the prime labeling of certain classes of graphs like $\mathrm{H}$-graph,Gear graph,Sunflower graph and corona of $\mathrm{C}_{\mathrm{n}}$ and $\mathrm{k}_{1,3}$ It is very difficult to generalize these labeling due to the nature the prime numbers.It is of interest to look in to certain kind of graphs where in total prime labeling is possible up to a large prime and verify this by programming concepts.

\section{REFERENCES}

[1] Gallian, J.A. 2009. A Dynamic Survey of Graph Labeling, The Electronic Journal of Combinatorics, (Jan 31, 2009)

[2] Vasanthi, B. Sattanathan, R. 2011. Graceful labeling of Sun flower graphs, Proc. Int. Conf. Math. Comp. Sci., 533-536.

[3] Lee, S.M. Wue, I. and Yeh, J. (1988). On the amalgamation of prime graphs, Bull Malaysian Math. Soc., (2)11, 59-67.

[4] Annamma, V. (2010). Total vertex irregularity strength of H Graph and Gear graph, Proc. Int. Conf. Math. Comp. Sci., 237-239.

[5] Ramya, N. Rangarajan, K. and Sattanathan, R. (2011). On coloring of Bimagic 4-regular grphs, Int. Journal of Ultra Scientist of Physical Sciences, 23(3).

[6] Ramya, N. Rangarajan, K. and Sattanathan, R. 4-regular graphs and its Prime labeling, IJCGTA, accepted for publication

[7] Ramya, N. Rangarajan, K. and Sattanathan, R. (2012). Edge magic labeling of Some graphs, Proc. Nat. Conf (NCMTA-2012), 258-262.

[8] Ramya, N. Rangarajan, K. and Sattanathan, R. (2012), Edge magic and Anti magic labeling of Regular graphs, Proc. of ICMEB-2012.

[9] Seoud.M.A,Youssef.M.S, Harmonious labeling of helms and related graphs,unpublished.

[10] Indra Rajasingh, BharatiRajan and Helda Mercy,Exact Wirelength of $\mathrm{H}$ Graphs on Path, Mathematics and Computer Science(2009). 